\title{
Characteristics and Features of Electroencephalography (EEG) in Children with Epilepsy
}

\author{
Putu Tarita Susanti, I Gusti Ngurah Made Suwarba*, Dewi Sutriani Mahalini
}

Department of Child Health, Faculty of Medicine, Udayana University, Denpasar, Indonesia

\author{
Email address: \\ suwarbangurah@yahoo.co.id (I G. N. M. Suwarba) \\ ${ }^{*}$ Corresponding author
}

\section{To cite this article:}

Putu Tarita Susanti, I Gusti Ngurah Made Suwarba, Dewi Sutriani Mahalini. Characteristics and Features of Electroencephalography (EEG) in Children with Epilepsy. Clinical Neurology and Neuroscience. Vol. 4, No. 4, 2020, pp. 76-81. doi: 10.11648/j.cnn.20200404.12

Received: October 14, 2020; Accepted: October 23, 2020; Published: November 4, 2020

\begin{abstract}
Epilepsy is a manifestation of impaired brain function with various etiologies in the form of recurrent paroxysmal seizure symptoms. Epilepsy occurs mostly in children. Electroencephalography (EEG) is performed to observe epileptogenic foci, certain epilepsy syndromes, evaluate treatment, and determine prognosis. The purpose of this study is to determine the characteristics and features of EEG in pediatric patients with epilepsy at Sanglah General Hospital. This study is a retrospective descriptive observational study. The data was achieved from medical records of patients who had just been diagnosed with epilepsy at the Pediatric Clinic of Sanglah General Hospital during the periode of January 2017 to December 2019. There was a total of 204 new epilepsy cases, with the prevalence of 6.4 per 1000 population. The data consists of the men vs female ( $57.4 \%$ vs $42,6 \%)$ and the majority of patients age range between $1-5$ years old (49\%). The data consists of generalized epilepsy with motor tonic clonic onset (75.5\%) and focal epilepsy with motor tonic onset (20.6\%). EEG examination was abnormal in 55.4\% of the cases and abnormalities was found in head CT scans (42.7\%). The etiology of pediatrics with epilepsy which was unknown (52.5\%). More than half of anti-epileptic drug therapy used a monotherapy of valproic acid (54.4\%), and polytherapy therapy of valproic acid and carbamazepine (17.6\%). Most of the children had good nutritional status (76\%). The data showed that a history of neonatal seizures in was found in 17 cases (8.3\%), while a family history of epilepsy was found ini 34 cases (16.7\%). Conclution that pediatric epileptic patients present with a generalized type of epilepsy onset tonic-clonic motor with an unknown etiology. EEG images are mostly abnormal. On CT scans of the head, abnormalities were found in $42.7 \%$ of cases. Most of them received monotherapy with valproic acid.
\end{abstract}

Keywords: Epilepsy, Electroencephalography, Head Imaging, Characteristics, Children

\section{Introduction}

Epilepsy derives comes from the Greek word "Epilambanmein" which means as an attack. Epilepsy is a manifestation of impaired brain function with various etiologies, in the form of recurrent seizure attacks due to excessive and paroximal discharges of brain neurons. Seizures are the most common neurological disorder in children, with $4-10 \%$ of children having at least one seizure in the first 16 years of their life. Studies have shown that 150.000 children have seizures each year, of which 30.000 develop epilepsy. The incidence of epilepsy in children in developing countries is approximately 40 cases per 100.000 children each year. The Word Health Organization (WHO) estimates the prevalence (both new and old cases) of epilepsy requiring treatment at around 8.2 per 1000 world population; $80 \%$ of them are in developing countries. The peak prevalence of epilepsy is found in early adolescence to young adulthood and is most commonly seen at the age of under 1 years of age [1-3].

There are two categories of epileptic seizures, namely generalized and focal seizures. Focal seizures occur because of a lesion in one part of the cerebral cortex, which can be accompanied by partial loss of consciousness. While generalized seizures occur due to a lesion which occupies a large area of the cerebral cortex, and usually affects both cerebral hemispher $[4,5]$.

The causes of epilepsy are very diverse, including central nervous system (CNS) infections, head trauma, tumors, degenerative diseases, and metabolic diseases, but in about $60 \%$ of the cases there is no definite cause. Based on gender, it 
was found that the incidence of epilepsy in boys was higher than girls [6].

The main therapy for epilepsy is the administration of anti-epileptic drugs (AEDs) to control seizures. Around 50\% of seizure activity and recurrence frequency of patients with epilepsy can be controlled with AEDs, but 30-40\% still have difficulty to control the seizures even with AED. The treatment of epilepsy with AED is individual and distinctive, different from therapy of other diseases. This characteristic is associated with long durations of treatment $[7,8]$.

This study aims to determine the characteristics and features of electroencephalographic (EEG) of children with epilepsy at Sanglah General Hospital. From this study, the authors hope to provide information to increase knowledge and development of pediatric medicine regarding epilepsy in children.

\section{Material and Method}

This study use retrospective descriptive observational study. The data was achieved from medical records of pediatric patients with epilepsy in the Neurology Division of the Departement of Child Health, Medical Faculty of Udayana University / Sanglah General Hospital in Denpasar. The study was conducted by taking samples from outpatients at the children's polyclinic during January 2017 until December 2019 or until the minimum sample size was met. The sample was determined by taking medical record data using probability sampling in the Medical Record Instalation of Sanglah General Hospital in Denpasar.

Inclusion criterias were children between the age of 1 month to 18 years of age, who has been diagnosed with epilepsy and given AED therapy in the Neurology Division Polyclinic of Sanglah General Hospital. While exclusion criterias were those who had a lack in follow and incomplete medical records.

The sample size wa calculated using descriptive study formula, with the prevalence of epilepsy in children was $25 \%$, the determination of accuracy was $10 \%$, and $\mathrm{Z} \alpha$ was 1.96 , and a minimum sample was 72 subjects.

Data analysis was performed using SPSS software to describe the characteristics of subject and variables. Categorical variables are presented in terms of numbers and percentages. Data is presented in narrative and table form. This study has obtained ethical clearance from the Research Ethics Commission of the Faculty of Medicine, Udayana University / Sanglah General Hospital.

\section{Result}

The study collected as many as 204 newly diagnosed epilepsy cases with a prevalence of epilepsy ranging from 6.3 to 6.4 per 1000 population. Age onset was found in the 1-5 years of age group with a total of 100 cases $(49.0 \%)$. A comparision by gender showed that there were more male patients with a total of 117 cases $(57.4 \%)$. Most subjects had good nutritional status 155 cases $(76 \%)$. The data showed that a history of neonatal seizures in was found in 17 cases $(8.3 \%)$, while a family history of epilepsy was found ini 34 cases $(16.7 \%)$. Data on the characteristics of subjects in this study are shown in Table 1.

Table 1. Characteristic of Subjects.

\begin{tabular}{ll}
\hline Characteristic & N=204 \\
\hline Sex & $117(57.4)$ \\
Male, N (\%) & $87(42.6)$ \\
Female, N (\%) & \\
Age & $53(26.0)$ \\
$0-12$ months, N (\%) & $100(49.0)$ \\
$>1-5$ years, N (\%) & $51(25.0)$ \\
$>5$ years, N (\%) & \\
Nutritional Status & $6(2.9)$ \\
Obesity, N (\%) & $4(1.9)$ \\
Overweight, N (\%) & $155(76.0)$ \\
Well Nourished, N (\%) & $38(18.7)$ \\
Mild Malnutrition, N (\%) & $1(0.5)$ \\
Severe Malnutrition, N (\%) & \\
Neonatal Seizures & $17(8.3)$ \\
Yes, N (\%) & $187(91.7)$ \\
No, N (\%) & \\
Family history of epilepsy & $34(16.7)$ \\
Yes, N (\%) & $170(83.3)$ \\
No, N (\%) & \\
\hline
\end{tabular}

Generalized epilepsy was the most common type of epilepsy with 162 cases (79.4\%), consisting of 154 cases of tonic-clonic, 3 cases of tonic and 5 cases of absence seizures. On the other hand, there were only 42 cases $(20.6 \%)$ of focal epilepsy. Based on the etiologies, most cases were due to an unknown etiology $(52.5 \%)$, while other etiologies such as structural etiology was found in 38 cases $(18.6 \%)$, infection in 27 cases $(13.2 \%)$, genetic factor in 21 cases $(10.3 \%)$, metabolic in 6 cases $(2.9 \%)$ and immunological in 5 cases $(2.5 \%)$. Data on clinical features of pediatric epilepsy patients are presented in Table 2.

Table 2. Clinical Presentation in Children with Epilepsy.

\begin{tabular}{ll}
\hline Clinical Presentation & $\mathbf{N}=\mathbf{2 0 4}$ \\
\hline Type of Epilepsy (Generalized) & \\
Tonic clonic, N (\%) & $154(75.5)$ \\
Tonic, N (\%) & $3(1.4)$ \\
Absance, N (\%) & $5(2.5)$ \\
Type of Epilepsy (Focal) & \\
Tonic, N (\%) & $42(20.6)$ \\
Etiology & \\
Unknown, N (\%) & $107(52.5)$ \\
Structural, N (\%) & $38(18.6)$ \\
Infection, N (\%) & $27(13.2)$ \\
Genetic, N (\%) & $21(10.3)$ \\
Metabolic, N (\%) & $6(2.9)$ \\
Imunology, N (\%) & $5(2.5)$ \\
\hline
\end{tabular}

In diagnostic examinations, the first EEG showed normal features in 91 cases $(44.6 \%)$, while the total amount of cases with abnormal features I, II and III in the EEG were 16, 63 and 34, respectively. Head CT scan imaging was performed in 103 out of 204 cases $(57.3 \%)$, with abnormal results in only 44 cases (42.7\%). EEG and head CT scan data of pediatric patients with epilepsy are presented in Table 3. 
Table 3. EEG Features dan Head CT scan Characteristics in Children with Epilepsy.

\begin{tabular}{ll}
\hline Clinical Presentation & $\mathbf{N}(\%)$ \\
\hline EEG Features (N=204) & \\
Normal & $91(44.6)$ \\
Abnormal I & $16(7.8)$ \\
Abnormal II & $63(30.9)$ \\
Abnormal III & $34(16.7)$ \\
Head CT scan characteristics (N=103) & \\
Normal & $59(57.3)$ \\
Abnormal & $44(42.7)$ \\
\hline
\end{tabular}

The anti epileptic drug (AED) of choice for monotherapy was valproic acid (54.4\%), carbamazepine (15.2\%) and phenytoin/phenobarbital (3.4\%). Polytherapy of AED consists of 2 types of drugs, namely valproic acid in combination with carbamazepine (17.6\%), carbamazepine with phenytoin/phenobarbital (3.4\%) and valproic acid with phenytoin/phenobarbital (1.5\%). Meanwhile, polytherapy consisting of 3 drug combinations used valproic acid, carbamazepine and phenytoin/phenobarbital in 9 cases (4.5\%). Data on pediatric patients with epilepsy therapy are presented in Table 4.

Table 4. Therapy for Children with Epilepsy.

\begin{tabular}{ll}
\hline Type of Theraphy & N=204 \\
\hline Monotheraphy & $111(54.4)$ \\
Valproic acid, N (\%) & $31(15.2)$ \\
Carbamazepine, N (\%) & $7(3.4)$ \\
$\begin{array}{l}\text { Phenytoin/Phenobarbital, N (\%) } \\
\text { Politheraphy }\end{array}$ & $36(17.6)$ \\
Valproic Acid + Carbamazepine, N (\%) & $3(1.5)$ \\
Valproic Acid + Phenytoin/Phenobarbital, N (\%) & $7(3.4)$ \\
Carbamazepine + Phenytoin/Phenobarbital, N (\%) & $9(4.5)$ \\
Valproic acid + Carbamazepine + & \\
Phenytoin/Phenobarbital, N (\%) & \\
\hline
\end{tabular}

\section{Discussion}

Epilepsy is a paroxysmal event of temporary altered consciousness caused by brain dysfunction. Seizures can be epileptic due to excess electrical activity in the brain, or non-epileptic. Epilepsy is a clinical diagnosis, which is characterized by recurrent paroxysmal epileptic seizures for more than 24 hours and without any provocation. Syndrome epilepsy is epilepsy which is characterized by a group of clinical symptoms and signs that occur together including the type of attack, etiology, anatomy, trigger factors, age of onset, severity of disease, chronicity and prognosis [9-11].

A study in 2014 conducted by Baldin et al. found that children with seizure onset between the age of 1-19 years had a greater proportion of epileptiform waves than those aged under 1 years old, respectively $82.4 \%$ (HR 1.8; CI 95\% 1.3-2.3) and 72.8\% (HR 1.3; CI 95\% 0.9-2.2) [12]. Current developments in this field shows that the largest case of epilepsy in children had an onset under 5 years of age. This study shows the same results as previous studies where the onset mostly occurred between 1-5 years old (49.0\%).

Characteristics this study's subjects showed that epilepsy cases occurred more frequently in males (57.4\%). There are several epilepsy syndromes, with a greater prevalence in certain sexes. It is not yet certain the risk factors and causes, but it is suspected that sex hormones are related to the etiology of epilepsy [13]. Based on gender, males have a higher risk of suffering from epilepsy, but no racial differences were found. The incidence rate was higher in males in this study was similar to other studies in Turkey, where $59.3 \%$ of them were found to be a risk factor for epilepsy (OR 1.38; 95\% CI 1.13-1.69) [14].

The distribution of nutritional status in children with epilepsy mostly consists of good nutritional status (76.0\%). This is because in this study, the subjects were newly diagnosed cases of epilepsy with an unknown etiology, where most of them received adequate nutrition, even if there were nutritional disorders such as energy protein, mild to severe malnutrition, it would have been a case that had an underlying disease that affected oromotor which resulted in inadequate nutrition. Most cases of malnutrition that were found had structural etiologies from the central nervous system.

Immature brain tissue predisposes to seizures with the highest incidence in the first year of life [15]. At the age of less than 1 year, nerve cells are still immature, marked by the development of excitation canals $(\mathrm{Ca} 2+$ and $\mathrm{K}+$ ) preceding the development of inhibition pathways $(\mathrm{Na}+)$. Axon branching and excitation synapses also develop more rapidly so they are susceptible to excitation $[16,17]$. This is not in accordance with the data in this study where the incidence of neonatal seizures was found in only $8.3 \%$ cases. This is because the knowledge of the parents about the clinical occurrence of seizures at a neonatal age is very minimal so that no actual data could be obtained.

Genetics is believed to be involved in the majority of cases, either directly or indirectly. Some epilepsy can be caused by defects in a single gene (1-2\%); a large part is the result of the interaction of several genes and environmental factors. Some of the genes involved affect ion channels, enzymes, GABA, and $G$ protein-related receptors. In identical twins, if one person has epilepsy, there is a $50-60 \%$ chance that the other twin will also have epilepsy. In non-identical twins, the risk is $15 \%$. This risk is greater in patients with generalized seizures than in focal seizures. If both twins have epilepsy, they are more likely to have the same epilepsy syndrome (70-90\%) [18]. Other close relatives of people with epilepsy have a five times greater risk than those who do not. Between $1-10 \%$ of people with Down syndrome and $90 \%$ of people with Angelman syndrome have epilepsy [19]. This is not consistent with the data from this study, only $16.7 \%$ of cases had a family history of epilepsy. Several factors could influence this, namely race, culture and education and knowledge of parents. The stigma of epilepsy can also affect the patients' family.

Epileptic seizures are divided into focal (partial) seizures and generalized seizures depending on the focus and spread $[11,20]$. In focal seizures there is hyperexcitability in areas of neurons in the cortex that spread to the surrounding area through the corpus callosum or other commissures to the contralateral cerebral hemisphere or via subcortical pathways in partial seizures is becoming common. In generalized 
seizures there is direct hyperexcitability of both cerebral cortex and spread through the corpus callosum [21].

Generalized seizures had a greater proportion than focal seizures, namely $79.4 \%$ and $20.6 \%$, respectively. In this study, the general type of epilepsy was found to be epilepsy onset of motor tonic-clonic $(75.5 \%)$, absence $(2.5 \%)$ and tonic $(1.4 \%)$. Based on the type of focal epilepsy, it was found that the type of focal epilepsy was tonic (20.6\%). A systematic review by Mac et al. found a prevalence of generalized seizures upto $50 \%$ and focal seizures of $31-50 \%$ [22]. Another study conducted by Berg et al. results showed $45.4 \%$ of generalized seizures and $55.1 \%$ focal seizures [23]. The differences in the proportion of seizure types varies because different operational definitions that are used for generalized and focal seizures. That study used focal / general criteria based on the findings on the EEG, whereas the study by Mac et al. used a mixture of clinical criteria as well as the findings on the EEG. [22, 23] Other studies in the general population by Baldin et al. and Marsan et al. found no different proportions in the idiopathic epilepsy group with symptomatic epileptiform events, which was found more in the generalized seizure group than focal seizures $(p<0$ 0001) $[15,20]$.

The etiology of seizures studied in patients with epilepsy showed the highest number and percentage of etiologic seizures caused by unknown (52.5\%), structural $(18.6 \%)$, infection $(13.2 \%)$, genetics $(10.3 \%)$, metabolic $(2.9 \%)$ then immunology $(2.5 \%)$. Epilepsy unknown is the absence of structural lesions in the brain or neurological deficits, of which this etiology is the most common with an incidence of about $40 \%$ worldwide. The cause of the neuroanatomy or neuropathological abnormality is unknown. Unknown epilepsy occurs in infants, children, adolescents and young adults with normal brain MRI and no previous history of significant medical abnormalities. Structural epilepsy is associated with abnormalities in brain structures that indicate an underlying disease or condition, namely: developmental and congenital abnormalities, both genetic and acquired, for example: head injury, space-pressure lesions, circulatory disorders of the brain, toxic, and neurodegenerative disorders. Genetic etiology can be caused by the presence of genetic factors from the family, but not all of them are related to hereditary diseases. Metabolic etiology is associated with the presence of metabolic disorders such as uremia and vitamin B6 deficiency. Immunological etiologies result from immunological disorders, such as autoimmune encephalitis. The etiology of infection is caused by infection of the central nervous system (CNS) such as meningitis, encephalitis and tuberculosis (TB), which are correlated with structural etiology [24]. According to a study by Vozikis et al. patients with meningitis, have a four times higher risk of developing epilepsy [25].

EEG results can help determine the type of seizure and the type of epilepsy so that the selection of anti-epilepsy is more precise and also help determine the prognosis [26]. Normal EEG results can be found in children with brain disorders, and on the other hand, abnormal EEGs can be found in normal children $[11,27]$. Approximately $50 \%$ of patients with epilepsy show epileptiform waves on the first EEG recording. A study on children reported by Gilbert et al., showed that $34 \%$ of epileptiform waves were found on repeated EEG and $14.5 \%$ in first EEG ( $p=0.001$ ) [26]. The proportion of epileptiform waves in epilepsy patients is around $48 \%$. This is not much different from the findings in previous studies which stated EEG sensitivity ranges from $25-56 \%$. The meta-analysis study by Gilbert et al. found the sensitivity of epileptiform EEG in pediatric patients with epilepsy has a wide range, namely 33-91\%. Interpretations of abnormal EEG include: (1) abnormal I, namely the presence of brain hypofunction but no epileptiform waves found on the EEG, (2) abnormal II, namely the absence of symptoms of brain hypofunction in the presence of epileftiform waves on the EEG, and (3) abnormal III, namely the presence of symptoms of brain hypofunction accompanied by epileptiform waves on EEG [28]. The results of the EEG may differ because getting a positive result could require several activation procedures, for example sleep, hyperventilation, and photic stimulation [29]. Other factors that could influence the outcome are the different readers can result to different interpretations (inter-observer bias). In this study, the results of normal EEG examination were found in $44.6 \%$ cases and abnormal EEGs being abnormal II (30.9\%), Abnormal III (16,7\%) and abnormal I (7,8\%).

The timing of the EEG recording may have an influence on the occurrence of epileptiform waves. The closer the distance between seizure events, the more likely the EEG will still be able to record epileptic processes in the brain. Studies according to Marsan et al. found an increase in the ratio of the occurrence of epileptiform waves on 1-3 days since the onset of seizures and decreases after day 4 [20]. A study by King et al. with 300 subjects (pediatric and adult) found epileptiform waves in $51 \%$ subjects when a EEG was performed within 24 hours of the seizures onset and in $34 \%$ with those who had an EEG thereafter [30]. The study by Narayanan et al. showed that had a seizure proportion of $89 \%$ in subjects who had an EEG within 24 hours of the seizures and none in subjects with EEG performed after 72 hours [31].

The waking state can render an EEG full of artifacts due to muscle movement [32]. Sleep during EEG recording can occur spontaneously, with deprivation, and the use of sedation. Spontaneous sleep during EEG recording is preferred over sedated sleep [33].

Imaging examinations that can be done are plain radiographs of the head, cerebral angiography, computed tomography scan, magnetic resonance imaging and positron emision tomography (PET). A CT scan of the head can detect some structural abnormalities of the brain such as focal calcifications, while a head MRI can better see abnormalities in the brain, especially abnormalities in the brain parenchyma. A study states that in pediatric patients with epilepsy, CT scan results are abnormal in about $7 \%-24 \%$ of cases, while abnormal head MRI was found in almost $50 \%$ of focal or partial epilepsy [34]. According to Rallam, CT scans show normal results in most cases of epilepsy [35]. This is similar to the results of this study, where normal CT scans were found in $57.3 \%$ of the subjects while abnormal in $42.7 \%$. 
Valproic acid has long been known effective in suppressing epileptiform waves in generalized, focal and absent epilepsy. The study by Bruni et al. showed clinical improvement in $75 \%$ of epilepsy patients, with $57 \%$ of patients showing reduction in nail-wave waves [36]. Glauser et al. showed that the AED available for monotherapy (phenytoin, carbamazepine, and valproate acid) in general still proved effective for seizure control in epilepsy patients, both in the adult and children, and in partial and general seizures. In certain conditions, if monotherapy was unable to prevent recurrent seizures, polytherapy can be given considering the profile of the drugs to be combined [37]. In this study, it was found that $54.4 \%$ of the subjects use valproic acid as a monotherapy therapy. This is because in this study the most common types of epilepsy found was generalized ones, hence the choice of monotherapy was valproic acid.

This study is large-scale at a central hospital so that it can produce general explanations as objective comparisons at different times and places.

Limitation of our study was a descriptive study based on medical record data, so there were many shortcomings and weaknesses, it is recommended to conduct prospective research to obtain more precise data.

\section{Conclusion}

This study shows that the prevalence of 6.4 per 1000 population. Most of them with unknown etiology and have abnormal EEG examination. Head CT scan was conducted in 103 from 204 cases with abnormalities found in $42.7 \%$ of them. The most common generalized epilepsy was tonic clonic, with monotherapy drug, valproic acid.

\section{Disclosure}

All the authors do not have any possible conflicts of interest.

\section{Acknowledgements}

None declared.

\section{References}

[1] Fisher, R. S., Boas, W. V. E., Blume, W., Elger, C., Genton, P., Lee, P., et al. Epileptic Seizures and Epilepsy: Definition Proposed by the International League Against Epilepsy (ILAE) and the International Bureau for Epilepsy (IBE). Epilepsia. 2005; 46 (4): 470-2.

[2] McAbee, G. N., Wark, J. E. A practical approach to uncomplicated seizures in children. Am Fam Physician. 2000; 62 (5): 1109-16.

[3] Mitchell, W. G., Hirtum, M. V., Desai, J., Luc, Q. N., Behavior, M. V., Desai, J., et al. Behavioral, cognitive and social aspect of childhood epilepsy. $5^{\text {th }}$ Edition. USA: Mosby Elsevier. 2012: p. 871-9.
[4] Guidelines for epidemiologic studies on epilepsy. Commission on Epidemiology and Prognosis, International League Against Epilepsy. Epilepsia. 1993; 34: 592-6.

[5] Engel, J. Jr. A Proposed Diagnostic Scheme for People with Epileptic Seizures and with Epilepsy: Report of the ILAE Task Force on Classification and Terminology. Epilepsia. 2001; 42 (6): 796-803.

[6] Annegers, J. F., Rocca, W. A., Hauser, W. A. Cause of epilepsy: contributions of the Rochester epidemiology project. Mayo Clin Proc. 1996; 71 (6): 570-5.

[7] Brodie, M. J., French, J. A. Management of epilepsy in adolescents and adults. Lancet. 2000; 22; 356 (9226): 323-9.

[8] Gidal, B. E., and Garnett, W. R. Epilepsy in Dipiro, Pharmacotherapy : A Pathophysiologic Approach. $6^{\text {th }}$ edition. USA: Mc. Graw Hill. 2005; p. 1023-48.

[9] Commission on Clasification and Terminology of the International League Against Epilepsy. Proposal for revised clinical and electrographic classifation of epileptic seizures. Epilepsia. 1981; 22: 489-501.

[10] Jimmy, P. 1996. Epidemiologi epilepsi. Buku Ajar Neurologi Anak. Jakarta: IDAI. 1996; h. 190-7.

[11] Commission on Epidemiology and Prognosis of the International League Against Epilepsy. Guidelines for epidemiologic studies of epilepsy. Epilepsia. 1993; 34: 592-6.

[12] Baldin, E., Hauser, W. A., Buchhalter, J. R., Hesdorffer, D. C., Ottman, R. Yield of epileptiform electroencephalogram abnormalities in incident unprovoked seizures: a population-based study. Epilepsia. 2014; 55: 1389-98.

[13] Christensen, J., Kjeldsen, M. K., Andersen, H., Friis, M. L., Sidenius, P. Gender differences in epilepsy. Epilepsia. 2005; 46: 956-60.

[14] Ali, C., Ayse, S., Deniz, Y., Vehbi, D., Secil, O., et al. Prevalence of some risk factors in childhood with epilepsy compared to their controls. Seizure. 2007; 16: 338-44.

[15] Stafstrom, C. E. The pathophysiology of epileptic seizure: a primer for pediatricians. Pediatr Rev. 1998; 19: 342-51.

[16] Shorvon, S. The concept of symptomatic epilepsy and the complexities of assigning cause in epilepsy. Epilepsy behave. 2014; 31: $1-8$

[17] Holmes, G. H. Epilepsy in the developing brain: lessons from the laboratory and clinic. Epilepsia. 1997; 38: 12-30.

[18] Pandolfo, M. Genetics of epilepsy. Semin Neurol. 2011; 31 (5): 506-18.

[19] Bhalla, D., Godet, B., Druet-Cabanac, M., Preux, P. M. Etiologies of epilepsy: a comprehensive review. Expert Rev Neurother. 2011; 11 (6): 861-76.

[20] Marsan, C. A., Zivin, L. S. Factors related to the occurrence of typical paroxysmal abnormalities in the EEG records of epileptic patients. Epilepsia. 1970; 11: 361-81.

[21] Pillai, J., Sperling, M. R. Interictal EEG and the diagnosis of epilepsy. Epilepsia. 2006; 47: 14-22.

[22] Mac, T. L., Tran, D. S., Quet, F., Odermatt, P., Preux, P. M., Tan, C. T. Epidemiology, etiology, and clinical management of epilepsy in Asia: a systematic review. Lancet Neurol. 2007; 6: 533-43. 
[23] Berg, A. T., Shinnar, S., Levy, S. R., Testa, F. M. Newly diagnosed epilepsy in children: presentation at diagnosis. Epilepsia. 1999; 40: 345-47.

[24] Robert, S., Helen, C., Carol, D., Jacquelline, A., Sheryl, R., et al. Instruction manual fot the ILAE 2017 operational classification of seizure types. Epilepsia. 2017; 58 (4): 531-42.

[25] Vozikis, A., Goulionis, J. E., Nikolakis, D. Risk Factors associated with epilepsy: a case-control study. Heal Sci J. 2012; 6 (3): 509-16

[26] Gilbert, D. L., Gartside, P. S. Factors affecting the yield of pediatric EEGs in clinical practice. Clin Pediatr. 2002; 41: 25-32.

[27] Eli, S., Jacob, G., Sarit, A., Aharon. The complementary of sleep devrivation on EEG. Eur J Paediatr Neurol. 2010; 12: 308-12.

[28] Gilbert, D. L., Sethuramana, G., Kotagal, U., Buncher, C. R. Meta-analysis of EEG test performance show wide variation among studies. Neurology. 2002; 60: 564-70.

[29] Deroos, S. T., Chillag, K. L., Keeler, M., Gilbert, D. L. Effect of sleep devrivation on the pediatric electroencephalogram. Pediatrics. 2009; 123: 703-8.

[30] King, M. A., Newton, M. R., Jackson, G. D., Fitt, G. J., Mitchell, L. A., Silvapulle, M. J., et al. Epileptology of the first-seizure presentation: a clinical, electroencephalographic, and magnetic resonance imaging study of 300 consecutive patients. Lancet. 1998; 352: 1007-11.

[31] Narayanan, J. T., Labar, D. R., Schaul, N. Latency to first spike in the EEG of epilepsy. Seizure. 2008; 17: 34-41.

[32] Fang, P. C., Chen, Y. J., Lee, I. C. Seizure precipitants in children with intractable epilepsy. Brain Dev. 2008; 30: 527-32.

[33] Michel, L. V., Jacques, M., Vincent, N., Paul, B., Michel, D. H., Claude, A., et al. Anticipation of epileptic seizures from standard EEG recordings. Lancet. 2001; 357: 183-7.

[34] Gaillar, W. D., Chiron, C., Cross, J. H., Harvey, S., Kuzniecky, R., et al. Guidelines for imaging infants and children with recent-onset epilepsy. Epilepsia. 2009; 50: 2147-53.

[35] Rallam DK. Investigating epilepsy: CT and MRI in epilepsy. Nepal Journal of Neuroscience. 2004: 67-72.

[36] Bruni, J., Wilder, B. J., Bauman, A. W., Willmore, L. J. Clinical efficacy and long-term effects of valproic acid therapy on spike-and-wave discharges. Neurology. 1980; 30: 42-6.

[37] Glauser, T., Ben-Menachem, E., Bourgeois, B. Updated ILAE Evidence Review of Antiepileptic Drug Efficacy and Effectiveness as Initial Monotherapy for Epileptic Seizures and Syndromes. Epilepsi. 2013; 54 (3): 251-61. 